\title{
EFECTO DEL REPOSO POST-TRANSFERENCIA EMBRIONARIA TRANSCERVICAL EN LA TASA DE EMBARAZO
}

\author{
Daisy Pezoa L. ${ }^{1}$, Juan Schwarze M. ${ }^{2}$, Yessenia Orellana A. ${ }^{2}$, Javier Arellano L. ${ }^{a}$, Juan \\ Pezoa L. ${ }^{b}$ \\ ${ }^{1}$ Médico Residente, Servicio de Salud Metropolitano Sur, Complejo Asistencial Barros Luco. ${ }^{2}$ Unidad de Medicina Re- \\ productiva, Clínica Las Condes.
}

${ }^{a}$ Internos de Medicina, Universidad de Santiago de Chile. ${ }^{b}$ Escuela de Medicina, Universidad San Sebastián.

\section{RESUMEN}

Antecedentes: Desde los primeros casos de fertilización in vitro se ha recomendado el reposo en cama posttransferencia embrionaria, para facilitar la implantación embrionaria y disminuir la posibilidad de expulsión. Esta recomendación, si bien común en todos los centros de reproducción asistida del mundo, no se basa en evidencia clínica. Objetivos: Buscar evidencia publicada respecto a la recomendación sobre el reposo posttransferencia y los mejores resultados de los ciclos de fertilización in vitro. Métodos: Se realizó una búsqueda sistemática de estudios publicados en inglés, alemán y español a través de Medline, Ovid y Biblioteca Cochrane. Resultados: Cinco artículos cumplieron los requisitos de inclusión y fueron considerados en el análisis. Si bien los artículos diferían en las comparaciones y tiempo de reposo, ninguno de ellos encontró una diferencia significativa a favor de realizar reposo post-transferencia. Conclusión: La evidencia publicada no relaciona el reposo post-transferencia con mejores resultados de ciclos de fertilización in vitro.

\section{PALABRAS CLAVES: Fertilización in vitro, transferencia embrionaria, reposo en cama}

\section{SUMMARY}

Background: Since first cases of in vitro fertilization bed rest has been recommended post-embryo transfer, in order to facilitate embryo implantation and reduce the possibility of its expulsion. This recommendation is common in all the assisted reproduction centers in rest of the world, however is not based on clinical evidence. Objectives: Search published evidence regarding the recommendation on the rest post embryo-transfer and best results of in vitro fertilization cycles. Methods: We conducted a systematic search for published studies in english, german and spanish through Medline, Ovid and Cochrane Library. Results: Five articles met the requirements for inclusion and were considered in this analysis. While articles differed in comparisons and resting time, none of them found a significant difference in favour of conducting rest post-transfer. Conclusion: The scientific evidence published does not relates rest post-transfer with best performing of in vitro fertilization cycles.

KEY WORDS: In vitro fertilization, embryo transfer, bed rest 


\section{INTRODUCCIÓN}

La transferencia embrionaria intrauterina es el último paso de las técnicas de reproducción asistida y puede determinar el resultado de las mismas $(1,2)$. Solamente una minoría de los embriones transferidos logrará implantarse y originar un embarazo. Distintas variables influyen en el éxito de este procedimiento, entre otros la calidad embrionaria, el uso de guía ecográfica y posición del catéter dentro del útero $(1,2)$.

Desde los primeros casos de reproducción asistida se ha insistido en el reposo post-transferencia por el temor de una eventual expulsión de los embriones depositados dentro del útero (3). Esta actitud, basada probablemente, en la noción que el reposo en cama es de bajo costo y no invasivo, pero que no tiene mayor evidencia que la sustente. El objetivo de esta revisión es determinar si la indicación de reposo post-transferencia embrionaria transcervical aumenta la tasa de embarazo en mujeres sometidas a técnicas de reproducción asistida.

\section{MÉTODO}

Realizamos una búsqueda bibilográfica en Pubmed, usando como criterio mesh "embryo transfer" y el keyword "rest". El mismo criterio se aplicó utilizando el motor de búsqueda de Ovid y en el sitio web de Cochrane. Se consideró como criterios de inclusión artículos publicados en los últimos diez años, escritos en inglés, alemán o castellano y con acceso al texto completo.

\section{RESULTADOS}

No encontramos ninguna revisión en Cochrane acerca del beneficio o la falta de beneficio del reposo post-transferencia transcervical de embriones en mujeres infértiles. La búsqueda con "embryo transfer" como mesh, arrojó un total de 9.517 artículos. Al limitarla con el uso de la palabra clave "rest", 31 artículos fueron encontrados. De ellos, 5 cumplieron los criterios de inclusión, por lo que fueron considerados en el análisis (Tabla I). La búsqueda en Ovid arrojó los mismos resultados.

Como se verá a continuación, los distintos estudios comparan distintos tiempos de reposo posttransferencia, lo que podría dificultar su interpretación. Sin embargo, ninguno de los estudios encontró diferencia en la tasa de embarazo al indicar reposo post-transferencia o al prolongarlo. Cabe destacar que en cada uno de los estudios se presentan los resultados según la tasa de embarazo clínico (con- firmado por ecografía o niveles de cuantitativos de gonadotrofina coriónica humana), con grupos de casos y controles comparables entre sí en cada estudio, en cuanto a edad, causas de infertilidad y número de embriones transferidos.

\section{DISCUSIÓN}

En 1997, el grupo del centro de medicina reproductiva de Birmingham, Reino Unido, publicó un estudio de caso control en una cohorte de 1.091 casos de ciclos de fertilización in vitro realizados dentro de un periodo de 26 meses (junio de 1994 a agosto de 1996) (4). Ellos compararon las tasas de embarazo con su protocolo de manejo, sin reposo post-transferencia de embriones, con todos los ciclos realizados en el Reino Unido en un periodo de 11 meses (abril de 1994 y marzo de 1995). Encontraron una mejor tasa de embarazo clínico en las pacientes que no fueron sometidas a reposo post-transferencia, 30\% versus $22,9 \%$, así como las tasas de embarazo por ciclo $23,5 \%$ versus $18,6 \%$ (variable no descrita en los siguientes estudios). Este ensayo clínico tiene la desventaja que no es controlado ni aleatorizado. En un intento de proporcionar algún tipo de comparación, los autores contrastaron los resultados de su centro con la de todos los ciclos similares en el Reino Unido. Por otra parte, la mayoría de las unidades de fertilización in vitro del Reino Unido practica el reposo en cama después de la transferencia de embriones. Por lo tanto, los autores proponen que esta comparación (con un gran número de ciclos involucrados), demuestra que el protocolo practicado, sin reposo post transferencia, no afecta las tasas de embarazo, incluso, las pacientes del estudio presentaban una mayor tasa de embarazo clínico que los controles, tanto por ciclo como por embrión transferido $(p=0,0001)$. Por lo que, debido a estos resultados favorables, sugieren que el reposo en cama no es necesario.

Ese mismo año, fue publicado un estudio multicéntrico realizado en Italia e Inglaterra (5), donde se comparó las tasas de embarazo de pacientes asignadas aleatoriamente en dos grupos, que eran homogéneos en cuanto a edad con una media de 31 años (rango: 20-42 años), duración y causas de infertilidad o el número de tratamientos previos. El primer grupo de 87 pacientes, se sometió a 97 ciclos de tratamiento, con 87 transferencias de embriones, seguida de un período de 24 horas de reposo en cama. En el segundo grupo 95 pacientes fueron sometidas a 102 ciclos de tratamiento y 93 realizaron reposo en cama posterior a la transferencia de embriones, por un período de 20 minutos. 


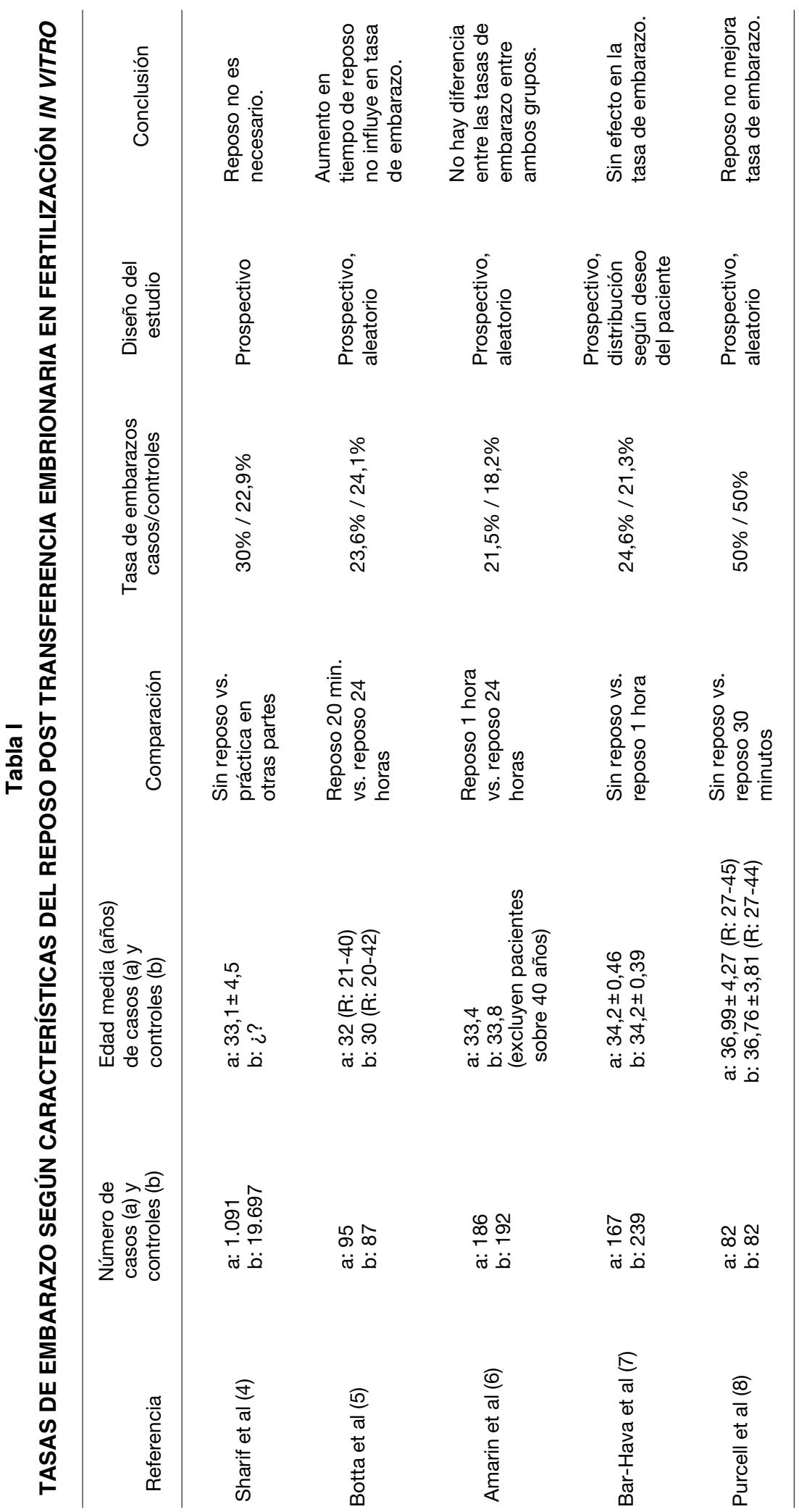


La tasa de embarazo por ciclo no difirió significativamente: $21,6 \%$ en el grupo con 24 horas de reposo y $21,3 \%$ en el grupo con 20 minutos de reposo. Este estudio muestra que un período de 24 horas de reposo en cama después de la transferencia de embriones no está asociado con un mejor resultado de la fertilización in vitro, en comparación con un período de descanso de 20 minutos. Este estudio tuvo la fortaleza que su diseño era prospectivo, que las pacientes fueron asignadas aleatoriamente a ambos grupos, y que ambos grupos tenían características demográficas y patológicas comparables.

En el 2004, un grupo de Arabia Saudita publicó los resultados de un nuevo estudio (6). Estudiaron en un período de 20 meses (mayo de 1999 a junio de 2001), 378 pacientes sometidas a fertilización in vitro fueron asignadas aleatoriamente a descansar por 1 hora (186 pacientes) o 24 horas (196 pacientes) una vez realizada la transferencia embrionaria. Se asignó a las pacientes a dos grupos con características semejantes, se excluyeron las pacientes mayores de 40 años, la edad media de las mujeres fue 33 años. Si bien no existió enmascaramiento por parte de las pacientes con respecto al tratamiento que recibieron, éstas ignoraban la existencia de un segundo grupo de estudio. Los resultados en ambos grupos fueron comparables, las tasas de embarazo clínico fueron $21,5 \%$ para los de 1 hora y $18,2 \%$ para las de 24 horas de reposo. La tasa por implantación de embriones fue significativamente mayor en el grupo de 1 hora de descanso $(14,4 \%)$ que en el de 24 horas ( $9 \%$ ). Sin embargo, 24 horas de descanso se traducen en una reducción de la tasa por implantación de embriones. El riesgo relativo de embarazo de los grupos con 1 hora y 24 horas de reposo fue de 1,11 con un intervalo de confianza de 0,87-1,41.

En 2005, un grupo de Israel publicó los resultados obtenidos durante el año 2001 (7). Se les ofreció a las 406 pacientes sometidas a fertilización in vitro durante este período, la posibilidad de no hacer reposo después de la transferencia de embriones o hacer reposo durante una hora. Ciento sesenta y siete pacientes prefirieron deambular inmediatamente después de la transferencia y 239 pacientes eligieron el reposo en cama. No hubo diferencias entre los grupos en las causas de la infertilidad. Por otra parte, no se encontraron diferencias estadísticamente significativas entre los grupos en edad del paciente, el número de ciclos de fertilización in vitro, ni otras variables estudiadas. Las tasas de embarazo no difirieron entre los dos grupos: $41(24,55 \%)$ en las con deambulación inmediata y $51(21,34 \%)$ en el grupo con 1 hora de reposo en cama. Concluyen que la deambulación inmedia- ta tras la transferencia de embriones, no tiene influencia negativa sobre la capacidad para concebir. Como se ve, el sesgo de ofrecer a las pacientes participar de acuerdo a su preferencia dificulta la interpretación de los resultados.

Finalmente, en 2007, fue publicado un estudio multicéntrico prospectivo, realizado en Estados Unidos de Norte América (8). En este estudio se reclutaron 152 pacientes a los cuales se les realizaron 164 ciclos de fertilización in vitro, distribuyéndose aleatoriamente en dos grupos de 82 cada uno; el primero con deambulación inmediata post-transferencia y el otro con treinta minutos de reposo en cama. La edad media para ambos grupos fue de 36 años (rango: 27-45 años). La tasa de embarazo clínico no difirió significativamente entre ambos grupos: $50 \%$ en el grupo con reposo y $50 \%$ en el grupo con deambulación inmediata. Se realizo un análisis logístico para introducir los ajustes correspondientes a otros factores conocidos que fueron estudiados, que afectan la tasa de embarazo, no afectando significativamente los resultados. Se concluye que el reposo por 30 minutos después de la transferencia de embriones no aumenta la tasa de embarazo. Este estudio tiene la fortaleza de ser un ensayo clínico aleatorizado y controlado, por lo que no existen los sesgos inherentes a otros tipos de estudios, pero llama la atención la alta tasa de embarazo en ambos grupos, a pesar de no haber diferencia estadísticamente significativa entre ambos, probablemente debido a una alta proporción de pacientes jóvenes con eventual mejor pronóstico, lo que no es analizado con más detalle en la discusión.

\section{CONCLUSIÓN}

La evidencia científica disponible no sustenta que el reposo post-transferencia embrionaria intrauterina transcervical, mejore la tasa de embarazo en pacientes sometidas a tratamientos de reproducción asistida. Esto permite autorizar a las pacientes a reanudar sus actividades inmediatamente posttransferencia, disminuyendo los costos relativos a días de hospitalización y horas de trabajo para las pacientes sometidas a este procedimiento.

\section{BIBLIOGRAFÍA}

1. Flisser E, Grifo JA. Is what we clearly see really so obvious? Ultrasonography and transcervical embryo transfer--a review. Fertil Steril 2007;1(87):1-5.

2. Abou-Setta AM, Mansour RT, Al-Inany HG, Aboulghar $\mathrm{MM}$, Aboulghar MA, Serour GI. Among women undergoing embryo transfer, is the probability of pregnancy and live birth improved with ultrasound guidance over clinical touch alone? A systemic review and meta- 
analysis of prospective randomized trials. Fertil Steril 2007;2(88):333-41.

3. Edwards RG, Fishel SB, Cohen J, Fehilly CB, Purdy $J M$, Slater JM, et al. Factors influencing the success of in vitro fertilization for alleviating human infertility. $J$ In Vitro Fert Embryo Transf 1984;1(1):3-23.

4. Sharif K, Afnan M, Lashen H, Elgendy M, Morgan C, Sinclair $\mathrm{L}$. Is bed rest following embryo transfer necessary? Fertil Steril 1998;3(69):478-81.

5. Botta G, Grudzinskas G. Is a prolonged bed rest following embryo transfer useful? Hum Reprod
1997;11(12):2489-92.

6. Amarin ZO, Obeidat BR. Bed rest versus free mobilization following embryo transfer: a prospective randomized study. BJOG 2004;11(111):1273-6.

7. Bar-Hava I, Kerner R, Yoeli R, Ashkenazi J, Shalev Y, Orvieto R. Immediate ambulation after embryo transfer: a prospective study. Fertil Steril 2005;3(83):5947.

8. Purcell KJ, Schembri M, Telles TL, Fujimoto VY, Cedars MI. Bed rest after embryo transfer: a randomized controlled trial. Fertil Steril 2007;6(87):1322-6. 